\title{
A SEARCH FOR MAGNETIC MONOPOLES WITH THE SOUDAN 2 DETECTOR*
}

W. W. M. Allison ${ }^{3}$, G.J. Alner ${ }^{4}$, I. Ambats ${ }^{1}$, D.S. Ayres ${ }^{1}$, L. Balka ${ }^{1}$, G.D. Barr ${ }^{3}$, W.L. Barrett ${ }^{1}$, D. Benjamin ${ }^{5}$, P. Border ${ }^{2}$, C. B. Brooks ${ }^{3}$, J. H. Cobb ${ }^{3}$, D.J.A. Cockerill $^{4}$, H. Courant ${ }^{2}$, B. Dahlin ${ }^{2}$, J. Dawson ${ }^{1}$, D. Demuth ${ }^{2}$, V.W. Edwards ${ }^{4}$, B. Ewen ${ }^{5}$, T. Fields ${ }^{1}$, L. M. Kirby-Gallagher ${ }^{3}$, C. Garcia-Garcia ${ }^{4}$, R. H. Giles ${ }^{3}$, G.L. Giller ${ }^{3}$,M.C. Goodman ${ }^{1}$, R. Gray ${ }^{2}$, S.J. Heilig ${ }^{1}$, S. Heppelmann ${ }^{2}$, N. Hill ${ }^{1}$, D.J. Jankowski ${ }^{1}$, K. Johns ${ }^{2}$, T. Joyce ${ }^{2}$,T. Kafka ${ }^{5}$, S. Kasahara ${ }^{2}$, J. Kochocki ${ }^{5}$, P. J. Litchfield $^{4}$, N. Longley ${ }^{2}$, F. Lopez ${ }^{1}$, M. Lowe ${ }^{2}$, W.A. Mann ${ }^{5}$, M.L. Marshak ${ }^{2}$, E.N. May $^{1}$, L. McMaster ${ }^{5}$, R. Milburn 5 , W.H. Miller ${ }^{2}$, C. Minor ${ }^{2}$, A. Napier ${ }^{5}$, W. Oliver ${ }^{5}$, G. F. Pearce ${ }^{4}$, D. H. Perkins ${ }^{3}$, E.A. Peterson ${ }^{2}$, L.E. Price ${ }^{1}$, D. Roback ${ }^{2}$, D. Rosen ${ }^{2}$, K. Ruddick ${ }^{2}$, B. Saitta ${ }^{5}$, D. Schmid ${ }^{2}$, J. Schlereth ${ }^{1}$, J. Schneps ${ }^{5}$, P. D. Shield ${ }^{3}$, M. Shupe ${ }^{2}$, N. Sundaralingam ${ }^{5}$, M. A. Thomson ${ }^{3}$, J.L. Thron ${ }^{1}$, G. Villaume ${ }^{2}$, L. Weems $^{2}$, S.J. Werkema ${ }^{2}$, N. West ${ }^{3}$ and C.A. Woods ${ }^{4}$

1. Argonne National Laboratory, Argonne IL 60439, USA;

2. University of Minnesota, Minneapolis MN 55455,USA;

3. University of Oxford, Oxford OX1 3RH, UK;

4. Rutherford Appleton Laboratory, Chilton, Didcot, Oxfordshire, OX11 OQX, UK; 5. Tufts University, Medford MA 02155, USA

\section{Abstract}

A search for GUT magnetic monopoles has been conducted using the Soudan 2 nucleon decay detector. This detector is a fine-grained tracking calorimeter. Monopole candidates were selected on the basis of significantly higher ionization than throughgoing cosmic ray muons. Preliminary results, using data taken over approximately one year with no monopoles observed, correspond to a flux limit of $2.410^{-14} \mathrm{~cm}^{-2} \mathrm{sr}^{-1} \mathrm{~s}^{-1}$ over a velocity range of $10^{-3}<\beta<0.95$.

Introduction The possible existence of magnetic charge was given a solid basis in 1931 when Dirac[1] showed that the existence of such monopoles was related to the quantization of electric charge. In 1974, t' Hooft[2] and Polyakov[3] showed that monopoles were a necessary consequence of the symmetry breaking which produces the electromagnetic group $U(1)$. This work also pointed to the likely miss of such monopoles, on the order of $\frac{M_{x}}{\alpha_{G U T}}$, or at least $10^{16} \mathrm{GeV}$. These monopoles would be highly penetrating, allowing their observation in underground ionization detectors. Numerous experimental searches have put increasingly stringent limits on the possible monopole flux. The flux limit reported here is the preliminary result from the Soudan 2 ionization detector.

The Detector The Soudan 2 detector is a large, underground, fine-grained, tracking calorimeter which is being built to search for nucleon decay. [4,5] It is located 2090 MWE below Soudan, MN, and will eventually have a mass of 1 kiloton. Tracks are detected by the ionization deposited in the $\mathrm{Ar}-\mathrm{CO}_{2}(85 \%-15 \%)$ gas. The ionization drifts out of a honeycomb array of resistive plastic tubes and is amplified and detected by a MWPC readout plane. Since the detector is 
modular, data are taken while installation is progressing. During this monopole search exposure it grew from $4.5 \times 5.4 \times 9.0 \mathrm{~m}^{3}$ to $9.0 \times 5.4 \times 9.0 \mathrm{~m}^{3}$. For each tube crossing along a track, all three spatial coordinates are recorded as well as the ionization. The detector has a spatial resolution of about $1 \mathrm{~cm}$ in all coordinates and usually records several hundred points per track, allowing it to produce bubble chamber like displays of events. These displays make it easy to scan events and pick out electromagnetic showers, multiple tracks, and other kinds of events.

Data Acquisiticn and Analysis All active elements of the detector participate in the trigger decision. The trigger configuration and threshold are set sufficiently sensitively to respond to local charge depositions from nucleon decays or atmospheric neutrino interactions; a single $150 \mathrm{MeV}$ kinetic energy muon causes a tigger more than $50 \%$ of the time. Throughgoing fast muon tracks easily satisfy this trigger, as would monopoles. The recorded events are about evenly divided between muon tracks and random coincidences of noise and/or radioactivity.

Since a fast monopole $\left(\beta>10^{-3}\right)$ ionizes increasingly heavily with velocity (from about 10 up to greater than 1000 times minimum ionizing)[6], the method used to find monopoles relies on finding tracks in the detector which have significantly higher ionization than the average muons. All the data are processed by an offine program which searches for tracks and writes out a condensed summary of the data for those tracks.[7] The summary contains, among other things, the track length and the summed ionization along the track.

A second pass program processes the summary data. It calculates a running average of the ionization per unit track length and flags tracks which have greater than four times this average ionization. The running average corrects for the effect of slowly changing conditions such as atmospheric pressure and gas quality. A cut requires the track length to be at least $1.5 \mathrm{~m}$. This assures that the track is long enough so that the average ionization along it is not unduly influenced by nonuniformities such as Landau fluctuations on individual hits or tracks which crossed the readout wire planes. A final cut is made on the fitting error of the points to the track to assure that the track is a stra: ght line.

For the 3140 events that were flagged by this filter, the raw data were retrieved from tape and scanned by a physicist using an interactive graphics program. The main criteria for rejection of tracks are based on the expectation that a monopole of GUT mass and $\beta<0.95$ will impart very little energy to an individual electron. In the Soudan 2 detector an electron will make an average of one hit per $15 \mathrm{MeV}$ of kinetic energy. [8] A delta ray of even 4 hits from an otherwise clean track can be easily identified. This would occur for a monopole with $\beta>0.95$ at which velocity it would be very heavily ionizing. Therefore, in order of frequency of cut application, the events were required not to have an associated shower, not to have an inelestic interaction, not to have any delta rays coming off the track and to be unifor nly highly ionizing. The last cut removed tracks passing through modules with excessive gas gain or traversing a long distance in the readout wire planes (which results in excessive charge amplification). Figure 1 shows a candidate event which passed most cuts but failed due to excessive wire plane amplification. No events passed all cuts.

In order to test the efficiency of this process for detecting monopoles, a set of 
simulated monopole tracks was created. This was done by taking real muon tracks which were selected tc be straight and noninteracting. A subset of these was then chosen which illuminated the detector isotropically, as sufficiently energetic monopoles would be expected to do. Raw data from these muon tracks were then altered to enhance the ionization by various factors. This was done only for the points that were part of the track. In addition, cross talk was added to neighboring channels and increasing tails were added to the pulses, to match what was observed with large pulses in the real data. Account was taken of the saturation of the ADC's in the read-out electronics. These simulated monopoie tracks were then inserted into the analysis chain to determine what fraction would be selected by oul software. Figure 2 shows the fraction of simulated monopole tracks which passed the ionization cutoff as a function of their ionization enhancernent factcr. These results can be used to determine the efficiency of the software nilter for detecting monopoles as a function of ionization. (E.g. for a factor of at least $10,90 \%$ of the simulated monopole tracks were selected by the filter.) The relationship between ionization and $\beta[14]$ allows us to express this efficiency as a function of $\beta$.

Exposure To determine the exposure of the detector both the spatial and the temporal acceptance of the detector are calculated. The geometry of the detector changes, in steps, as more modules are installed. For each configuration the areasolid angle product of the detector for isotropically arriving tracks was calculated by two methods. The first was a Monte Carlo approach which finds the cross-sectional area of the detector for randomly chosen directions, and the second is an analytical geometric calculation. During the time of the data taking the detector grew from 716 to $1124 \mathrm{~m}^{2} \mathrm{sr}$. These figures include the effect of the $1.5 \mathrm{~m}$ track length cut. The track length correction was determined from the fraction of simulated monopole tracks which failed the track length cut.

The duration of every data run (approximately 1.5 hours of data-taking) is recorded. For the data set which spanned 21-Jan-1989 to 31-Jul-1990, there were $2.5910^{7}$ live seconds. This is then corrected by the average dead time due to event read out, and unreadable tapes. The total exposure is calculated by summing the product of the corrected live time and the corrected geometric acceptance for each run. This gave an exposure of $1.210^{14} \mathrm{~cm}^{2} \mathrm{sr} s$.

Result The efficiency with respect to $\beta$ is multiplied by the exposure of the detector to provide an effective exposuse as a function of $\beta$. Since no monopoles were seen with this exposure, the resulting limit on the allowable monopole flux is $2.410^{-14} \mathrm{~cm}^{-2} \mathrm{~s}^{-1} \mathrm{sr}^{-1}$ for $10^{-3}<\beta<0.95$ (90\% confidence level). (See figure 3.)

\section{References:}

[1] P.A.M. Dirac, Proc. R. Soc. London Ser. A 133, 60 (1931).

[2] G. t' Hooft, Nuc. Phys. B 79, 276 (1974).

[3] A.M. Polyakov, JETP Lett. 20, 194 (1974).

[4] J. Thron et al., Nucl. Instr. Meth. A283, 642 (1989).

[5] D. Cockerill et al., to be published.

[6] S.P. Ahlen in "Magnetic Monopoles", ed. by R.C. Carrigan, Jr. and W.P. Trower (Plenum, New York, 1983) p. 259, and references therein.

[7] M. Lowe, Ph.D. Thesis, University of Minnesota, 1991.

[8] C. Garcia-Garcia, Ph.D. Thesis, University of Valencia, 1990. 

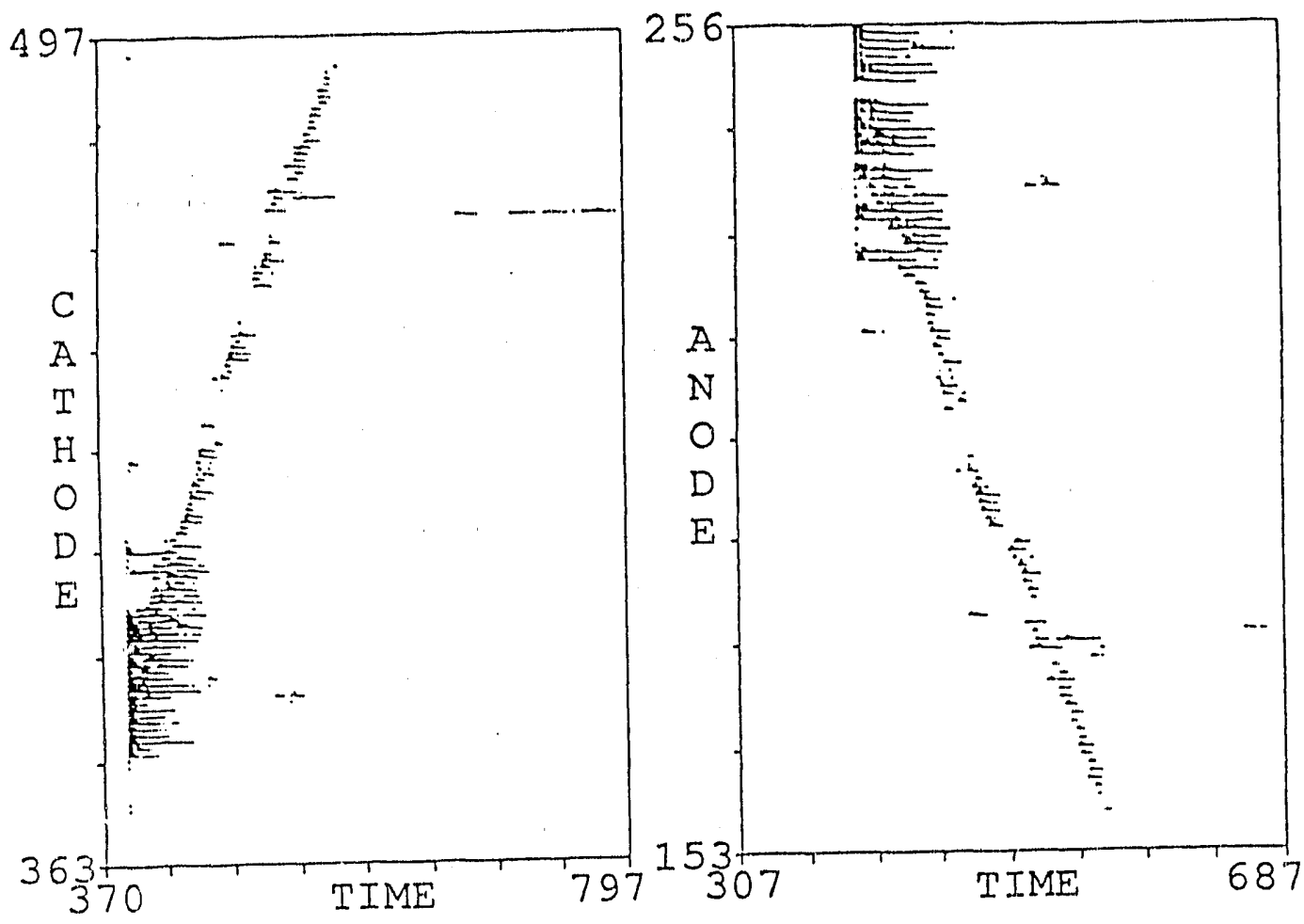

Figure 1. Run 12098, event 1701. Rejected due to excessive amplification at the endplane.
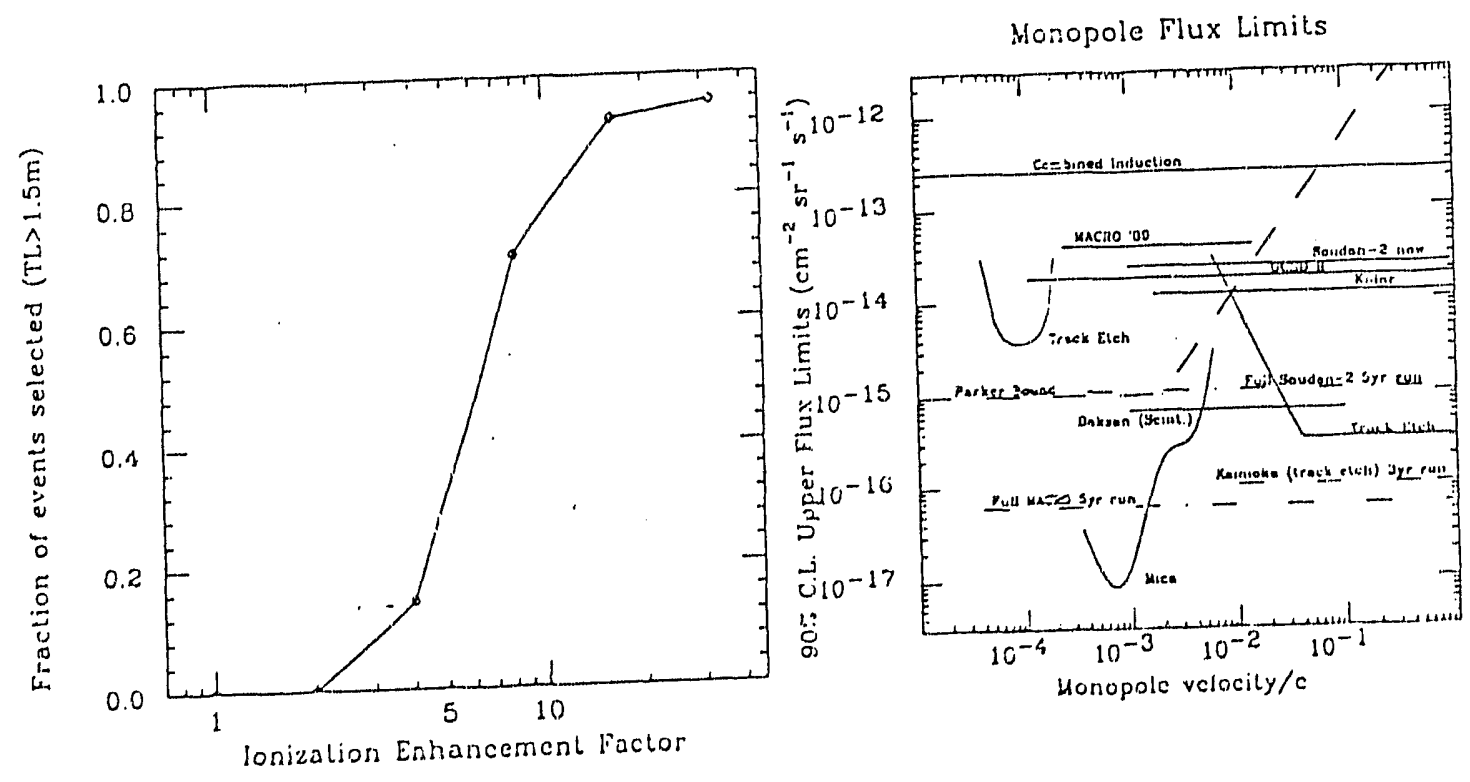

Figure 2. Fraction of fake monopoles passing Figure 3. Recent monopole flux limits. ionization cut vs. enhancement. 

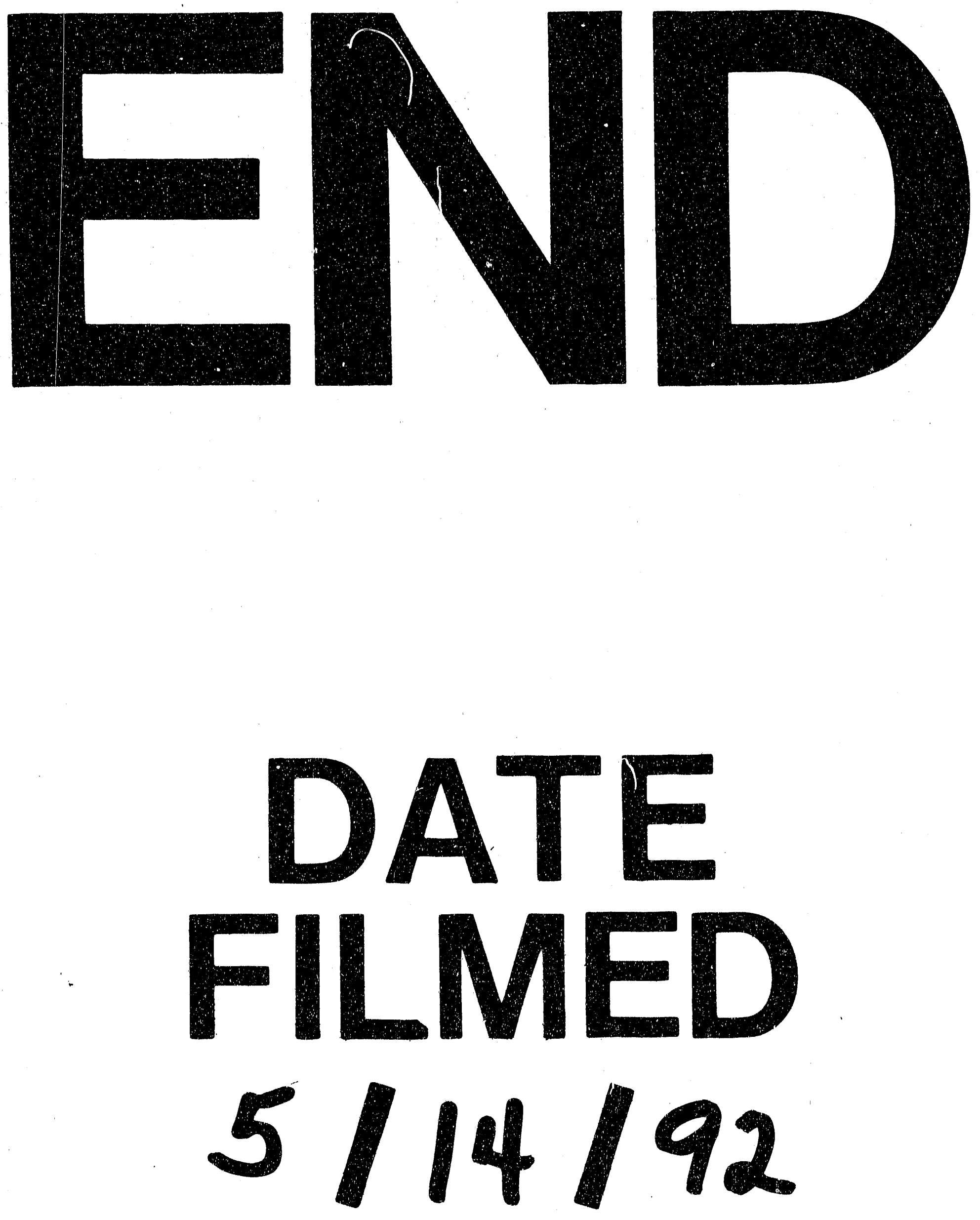

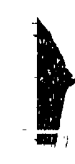


